\title{
Cardiac performance, biomarkers and gene expression studies in previously sedentary men participating in half-marathon training
}

\author{
Danica D Vance ${ }^{1,3+}$, Gordon L Chen ${ }^{3 \dagger}$, Mark Stoutenberg ${ }^{2,6}$, Robert J Myerburg ${ }^{3,4}$, Kevin Jacobs ${ }^{2}$, \\ Lubov Nathanson ${ }^{1}$, Arlette Perry ${ }^{2}$, David Seo ${ }^{1,5}$, Pascal J Goldschmidt-Clermont ${ }^{1,3}$ and Evadnie Rampersaud ${ }^{1,5^{*}}$
}

\begin{abstract}
Background: The mechanisms through which exercise reduces cardiovascular disease are not fully understood. We used echocardiograms, cardiac biomarkers and gene expression to investigate cardiovascular effects associated with exercise training.

Methods: Nineteen sedentary men (22-37 years) completed a 17-week half-marathon training program. Serial measurements of resting heart rate, blood pressure, maximum oxygen consumption, lipids, C-reactive protein, cardiac troponin T, echocardiograms and blood for gene expression were obtained from baseline to peak training. Controls included 22 sedentary men who did not exercise.

Results: Among the training group, $\mathrm{VO}_{2}$ max increased from 37.1 to $42.0 \mathrm{ml} / \mathrm{kg} / \mathrm{min}(\mathrm{p}<0.001)$. Significant changes were seen in left ventricular wall thickness and mass, stroke volume, resting heart rate and blood pressure $(p<0.001)$. The control group demonstrated no significant changes. Expression profiling in the training group identified 10 significantly over-expressed and 53 significantly under-expressed loci involved in inflammatory pathways. Dividing the training group into high and low responders based on percent change in $\mathrm{VO}_{2}$ max identified loci that differentiated these two groups at baseline and after training.

Conclusion: Intensive exercise training leads to significant increase in cardiac and hemodynamic performance, and significant changes in expression of genes involved in immune and inflammatory response.
\end{abstract}

Keywords: Gene expression, Exercise, Cardiovascular, Response

\section{Background}

Long-term exercise training has been associated with weight loss, improvements in lipid profile and blood pressure, and cardiac remodeling [1-3]. Despite this, the cardiovascular response to high-intensity training in previously untrained individuals is not well characterized. Importantly, even with extreme marathon and half-marathon (HM) exercise training, individuals may experience varying levels of cardiovascular and physiological changes, and these differences may have a biological basis. For example,

\footnotetext{
* Correspondence: ERampersaud@med.miami.edu

${ }^{\dagger}$ Equal contributors

'John P. Hussman Institute for Human Genomics, University of Miami, Miami, $\mathrm{FL}$, USA

${ }^{5}$ Dr. John T. Macdonald Foundation Department of Human Genetics, University of Miami, Miami, FL, USA

Full list of author information is available at the end of the article
}

HM and marathon training have been associated with increases in oxidative stress and myocardial damage [4-9]. HM and marathon training also induces the release of troponin I and T, markers of myocardial injury; elevations in cardiac troponin $\mathrm{T}(\mathrm{cTn} \mathrm{T})$ are reported in up to $39-56 \%$ of athletes immediately after performance [10]. Although the mechanism and implications are not well defined, these biomarkers usually return to normal within 24 to 48 hours, and the magnitude of biomarker peak is inversely related to the level of training prior to the event [10]. The purpose of our study was to assess time-related cardiovascular performance and function and corresponding gene expression changes in participants undergoing HM training. Our goal is to begin to identify a genomic basis for response to exercise by

\section{() Biomed Central}


correlating specific changes in gene expression with the physiologic changes following HM training.

\section{Methods}

Apparently healthy, untrained and sedentary males $(n=47)$, between 18 and 45 years of age, participated in the study. Candidates were required to have engaged in less than 2 hours of aerobic training per week prior to enrollment, have an average maximum oxygen consumption $\left(\mathrm{VO}_{2} \max \right)$ less than $50 \mathrm{ml} / \mathrm{kg} / \mathrm{min}$, and not previously completed a HM. Twenty-four of the subjects, who were enrolled in a local HM Training Program, were recruited into the training group (TRAIN), and twenty-three male subjects of similar age were recruited into the control group $(\mathrm{CON})$. The CON group did not participate in the training program or undergo any lifestyle modification over the 16 week duration of the study. All subjects volunteered for the study and signed informed consents. TRAIN subjects were compensated for meals, travel time, and training program fees. Exclusion criteria included smoking, known metabolic or cardiovascular conditions (diabetes or dyslipidemia), chronic inflammatory diseases, or chronic use of non-steroidal anti-inflammatory drugs. The protocol was approved by the University of Miami Institutional Review Board for human subjects research.

Initial screening and evaluation included a complete medical and training history, baseline $\mathrm{VO}_{2}$ max test, resting heart rate, blood pressure, height, weight, body composition assessment, serum biomarkers, and complete echocardiographic evaluation. Body composition was assessed through dual-energy X-ray absorptiometry scanning. Blood for the lipid panels and serum biomarkers was drawn via the antecubital vein using the LeukoLock Protection system and stored at $-80^{\circ} \mathrm{C}$ for analyses with the post-training RNA samples. Lipid panels were done on fasted blood samples. TRAIN and CON subjects had heart rate, blood pressure, height, weight, and serum biomarkers repeated at 6,11 , and 16 weeks after the baseline evaluation. Echocardiograms, $\mathrm{VO}_{2}$ max, and body fat evaluation were recorded at baseline and 16 weeks after the baseline evaluation.

Of the 24 subjects enrolled in the TRAIN group, 5 subjects were unable to complete the training due to injury or time constraints and withdrew prior to the peak training data (week 16) collection. Three subjects were able to complete the peak training data collection, but were unable to complete the HM due to injury. Thus, a total of 19 subjects completed the program with peak training data collected. Of these 19 subjects, 16 subjects completed the 2007 Miami HM and had blood drawn within 30 minutes after the race (see Figure 1). Of the $\mathrm{CON}$ group, one individual was unable to continue the study as a result of scheduling difficulty, leaving a total of 22 controls that completed all data collection.

Two-dimensional echocardiograms with pulsed-Doppler imaging were acquired on each subject using a commercial system (Philips HD11). Standard measurements were performed according to the American Society of Echocardiography guidelines [11]. Interventricular septal wall thickness, LV internal diameter, and LV posterior wall thickness were measured in systole and diastole in the parasternal long axis view. The LV end-diastolic volume, LV end-systolic volume, stroke volume, and ejection fraction were calculated via modified bi-planer calculation based on the apical 2 and 4 chamber view. LV mass was calculated using the area length method. With the pulsedDoppler trans-mitral filling velocities (peak early [E'] and late [atrial-A']), diastolic function was assessed and an E/A ratio was determined. One certified echocardiogram technician was responsible for acquiring all images for each participant. The measurements and calculations were independently confirmed by an experienced echocardiographer. Both were blinded to each subject's group status.

Blood was collected in EDTA and standard red top tubes (serum) and processed immediately. Tubes were immediately centrifuged, and the serum was separated and stored in a $-80^{\circ}$ freezer. Serum was analyzed for cTnT [12], CRP [12], and lipids (HDL, LDL, and TG) [12]. Blood

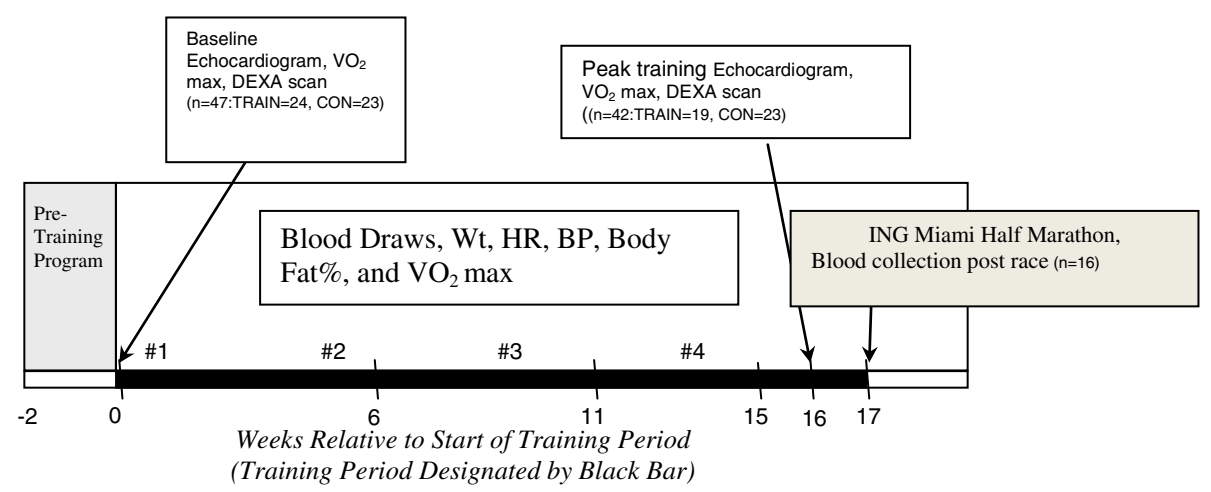

Figure 1 Study design. 
for RNA extraction was collected at both baseline and peak training (week 16) using the Ambion LeukoLOCK system that captured and preserved the blood mononuclear cell layer.

Clinical and biomarker data were analyzed using SPSS for Windows 15.0 software (SPSS, Inc, Chicago, IL). Frequency and descriptive statistics were calculated to check all relevant characteristics of the data. Independent samples $t$-tests were used to compare baseline variable differences between the TRAIN and CON groups. Paired samples $t$-tests were used to compare changes from baseline to peak training or baseline to post-HM.

Microarray analysis was performed on blood collected from each subject at baseline and at 16 weeks. Total RNA was extracted and analyzed using the Affymetrix GeneChip Human Gene ST array, which has 28,869 unique genes (or transcripts) which are captured by $\sim 26$ probes per gene. Data were normalized using Robust Multi-Array (RMA) Normalization. Significance Analysis of Microarray (SAM) Software [13] was used to determine statistically significant genes that met predetermined False Discovery Rate (FDR) thresholds. SAM uses non-parametric statistics and gene specific $t$-tests to identify statistically significant genes and to measure the strength of relationships between gene expression and a specific response variable. SAM also uses permutation tests to estimate the FDR after accounting for multiple testing corrections [14]. Genes with FDR $p$-values $<0.15$ and fold-change greater than 1.5 or less than -1.5 were considered significant. Pathway analysis was conducted using MetaCore ${ }^{\text {tm }}$ (GeneGo, Inc) software.

Two separate gene expression analyses were performed. The first compared differences in gene expression profiles between the CON and TRAIN subjects before and after the HM training. The second analysis focused only on the TRAIN subjects, by dividing them into high and low responders based on the percent change of their relative $\mathrm{VO}_{2} \max \left(\mathrm{rVO}_{2} \max \right)$ over the course of the training program. Relative $\mathrm{VO}_{2}$ max takes into account and individual's body weight and is defined as milliliters of oxygen per kilogram of bodyweight per minute. The mean percent change in $\mathrm{rVO}_{2}$ max was calculated and individuals who fell in the upper quartile (upper 25\%) of percent change in $\mathrm{rVO}_{2}$ max were labeled as highresponders (10 individuals) and those who fell below the upper quartile were considered low-responders $(9$ individuals). Gene expression differences between the two groups were studied at baseline and after the 16 weeks of training.

\section{Results}

Of the 16 subjects who completed the HM, the average time to completion was 2:22:21 (H:Min:Sec), $\mathrm{SD}=26: 33$. The fastest time was 1:39:00 and the slowest time was 3:11:58. No runner required medical attention after the race. There were no significant differences between the TRAIN and CON groups on any baseline characteristics other than triglycerides, which were significantly higher $(\mathrm{p}=0.03)$ in the TRAIN group (Table 1$)$.

Comparisons of basic physiological variables were made from baseline to the peak training time-point (17 weeks) in both groups (Table 1). $\mathrm{VO}_{2}$ max increased significantly $(\mathrm{p}=0.00004)$ among those in the TRAIN group during the course of the training program. In addition, the following were significantly decreased: (1) resting heart rate $(\mathrm{p}=0.002),(2)$ percent body fat $(\mathrm{p}=0.044)$, and (3) systolic $(\mathrm{p}=0.0001)$ and diastolic $(\mathrm{p}=0.007)$ blood pressure. Average body weight $(\mathrm{p}=0.08)$ and HDL $(\mathrm{p}=0.055)$ decreased slightly $(\mathrm{p}=0.08)$, but no other measured biomarkers (LDL, Tg, CRP, cTnT) showed significant improvements. There were no significant changes for any variables among the $\mathrm{CON}$ subjects (Table 1 ). Table 2

Table 1 Training group and control changes at baseline versus 16 weeks $(n=22)$

\begin{tabular}{|c|c|c|c|c|c|c|c|}
\hline \multirow[b]{2}{*}{ Variable } & \multicolumn{3}{|c|}{ Training group } & \multicolumn{3}{|c|}{ Control group } & \multirow[b]{2}{*}{ P value ${ }^{* *}$} \\
\hline & Baseline (M $\pm S D)$ & Peak $(M \pm S D)$ & P value* & Baseline (M $\pm S D)$ & Peak $(M \pm S D)$ & P value* & \\
\hline Weight & $196.76 \pm 28.40$ & $192.85 \pm 27.60$ & 0.08 & $188.31 \pm 26.11$ & $190.11 \pm 26.41$ & .064 & 0.316 \\
\hline Heart rate & $76.71 \pm 12.41$ & $67.60 \pm 11.76$ & 0.002 & $73.18 \pm 12.75$ & $76.05 \pm 15.99$ & .357 & 0.363 \\
\hline Systolic BP (mmHg) & $121.62 \pm 8.45$ & $114.30 \pm 9.23$ & 0.0001 & $116.67 \pm 9.86$ & $113.36 \pm 7.26$ & .133 & 0.088 \\
\hline Diastolic BP (mmHg) & $80.05 \pm 9.70$ & $73.15 \pm 8.20$ & 0.007 & $75.67 \pm 8.46$ & $77.14 \pm 5.20$ & .542 & 0.127 \\
\hline Relative $\mathrm{VO}_{2} \max$ & $37.06 \pm 7.12$ & $42.02 \pm 8.81$ & 0.00004 & $38.83 \pm 5.03$ & $39.02 \pm 6.74$ & .813 & 0.35 \\
\hline Body fat (\%) & $29.51 \pm 8.33$ & $28.18 \pm 8.86$ & 0.044 & $26.84 \pm 6.84$ & $27.45 \pm 6.87$ & .116 & 0.256 \\
\hline HDL & $46.71 \pm 9.61$ & $43.10 \pm 6.37$ & 0.055 & $49.59 \pm 7.87$ & $47.05 \pm 9.15$ & .183 & 0.288 \\
\hline LDL & $107.05 \pm 25.64$ & $103.80 \pm 28.03$ & 0.44 & $100.23 \pm 22.39$ & $105.86 \pm 20.61$ & .087 & 0.358 \\
\hline Triglycerides & $119.90 \pm 59.41$ & $116.35 \pm 72.01$ & 0.997 & $85.05 \pm 38.84$ & $77.68 \pm 40.87$ & .294 & 0.03 \\
\hline CRP & $1.92 \pm 2.66$ & $1.64 \pm 1.92$ & 0.752 & $0.84 \pm 0.66$ & $1.03 \pm 0.83$ & .269 & 0.084 \\
\hline Troponin $\mathrm{T}$ & $<0.01$ & $<0.01$ & 0.333 & $<0.01$ & $<0.01$ & .333 & 1 \\
\hline
\end{tabular}

*Difference in variable measures between baseline and peak. **Difference at baseline between Training and Control groups. 
Table 2 Echocardiographic changes over 16 weeks $(n=19)$

\begin{tabular}{|c|c|c|c|c|c|c|}
\hline \multirow[b]{2}{*}{ Variable } & \multicolumn{3}{|c|}{ Training group } & \multicolumn{3}{|c|}{ Control group } \\
\hline & Baseline $(\mathrm{M} \pm \mathrm{SD})$ & Peak (M $\pm S D)$ & $P$ value & Baseline (M \pm SD) & Peak $(M \pm S D)$ & $P$ value \\
\hline Interventricular septum in diastole $(\mathrm{cm})$ & $0.925 \pm 0.102$ & $1.003 \pm 0.128$ & 0.028 & $0.925 \pm 0.115$ & $0.982 \pm 0.138$ & 0.074 \\
\hline Left ventricular internal diameter in diastole $(\mathrm{cm})$ & $4.803 \pm 0.490$ & $4.818 \pm 0.490$ & 0.864 & $4.726 \pm 0.409$ & $4.876 \pm 0.451$ & 0.067 \\
\hline Left ventricular posterior wall in diastole $(\mathrm{cm})$ & $0.961 \pm 0.092$ & $1.075 \pm 0.123$ & 0.003 & $0.930 \pm 0.134$ & $0.963 \pm 0.133$ & 0.439 \\
\hline Left ventricular mass ( $\mathrm{g}$ ) & $186.6 \pm 37.4$ & $209.2 \pm 30.0$ & 0.009 & $183.0 \pm 31.1$ & $192.1 \pm 32.2$ & 0.114 \\
\hline Left ventricular mass index $\left(\mathrm{g} / \mathrm{m}^{2}\right)$ & $91.4 \pm 16.8$ & $102.8 \pm 12.8$ & 0.008 & $90.4 \pm 15.1$ & $94.2 \pm 14.1$ & 0.194 \\
\hline Left ventricular end-diastolic volume (ml) & $118.4 \pm 23.8$ & $144.1 \pm 29.6$ & 0.002 & $124.7 \pm 32.7$ & $133.1 \pm 30.8$ & 0.186 \\
\hline Left ventricular end-systolic volume (ml) & $45.8 \pm 16.4$ & $52.4 \pm 16.7$ & 0.195 & $43.4 \pm 14.0$ & $48.0 \pm 14.8$ & 0.11 \\
\hline Ejection fraction (\%) & $61.9 \pm 7.9$ & $63.8 \pm 7.8$ & 0.461 & $65.2 \pm 6.5$ & $64.2 \pm 6.5$ & 0.47 \\
\hline Stroke volume (ml) & $72.6 \pm 14.0$ & $91.6 \pm 20.9$ & 0.001 & $81.2 \pm 22.3$ & $85.1 \pm 20.4$ & 0.411 \\
\hline Peak early to late mitral annular velocities (E to A ratio) & $1.59 \pm 0.37$ & $1.38 \pm 0.40$ & 0.03 & $1.67 \pm 0.43$ & $1.70 \pm 0.48$ & 0.766 \\
\hline
\end{tabular}

Table 1 shows comparison of echocardiographic changes between TRAIN and CON groups. The following significant changes occurred in the TRAIN group: (1) interventricular septal thickness increased from $0.925 \pm 0.102 \mathrm{~cm}$ to $1.003 \pm 0.128 \mathrm{~cm}(\mathrm{p}=0.028)$, (2) posterior wall thickness in diastole increased from $0.961 \pm 0.092 \mathrm{~cm}$ to $1.075 \pm 0.123 \mathrm{~cm}(\mathrm{p}=0.003)$, (3) LV mass increased from $186.6 \pm 37.4 \mathrm{~g}$ to $209.2 \pm 30.0 \mathrm{~g}$ ( $p=0.009),(4) \mathrm{LV}$ mass index increased from $91.4 \pm 16.8 \mathrm{~g} / \mathrm{m}^{2}$ to $102.8 \pm 12.8 \mathrm{~g} / \mathrm{m}^{2}(\mathrm{p}=0.008)$, (5) LV end diastolic volume increased from $118.4 \pm 23.8 \mathrm{ml}$ to $144.1 \pm 29.6 \mathrm{ml}$ ( $\left.p=0.002\right)$, (5) stroke volume increased from $72.6 \pm 14.0 \mathrm{ml}$ to $91.6 \pm 20.9 \mathrm{ml}(p=0.001)$, and (6) the $E$ to $A$ ratio decreased from $1.59 \pm 0.37$ to $1.38 \pm 0.40(p=0.03)$. No significant changes occurred within the control group from baseline to 16 week follow-up.

shows comparison of echocardiographic changes between TRAIN and CON groups.

In comparing the gene expression profiles between TRAIN and CON individuals before training, only 4 genes (CMKLR1, FOS, IFIT1, and PDK4) showed significant changes $(\mathrm{FC}>1.5, \mathrm{FDR}<0.05)$ (See Table 3$)$. All had

Table 3 Genes with significantly increased expression levels in active (TRAIN) vs. sedentary (CON) individuals (FDR <0.05)

\begin{tabular}{ccc}
\hline \multicolumn{2}{c}{ Before marathon training - Increased gene expression* } \\
\hline Transcript id & Gene & Fold change (FC) \\
\hline 7966089 & CMKLR1 & 1.56 \\
7975779 & FOS & 1.74 \\
7929065 & IFIT1 & 1.51 \\
8141094 & PDK4 & 1.53 \\
\hline
\end{tabular}

\begin{tabular}{ccc}
\hline \multicolumn{2}{c}{ After marathon training - increased gene expression* } \\
\hline Transcript id & Gene & Fold change (FC) \\
\hline 7929256 & - & 1.6 \\
7928306 & - & 1.64 \\
7966089 & CMKLR1 & 1.68 \\
7906475 & FCRL6 & 1.66 \\
7996081 & GPR56 & 1.55 \\
7906764 & HSPA6 & 1.53 \\
7964787 & IFNG & 1.66 \\
8054722 & IL1B & 1.71 \\
7961175 & KLRC3 & 1.64 \\
7971661 & MIR15A & 1.8 \\
\hline
\end{tabular}

*FDR $=$ false discovery rate $(p<0.05$ threshold used). increased levels of gene expression in the TRAIN group compared to the CON group. No genes were significantly decreased.

When comparing profiles from blood collected in TRAIN and CON individuals after the HM training program, ten genes (CMKLR1, FCRL6, GPR56, HSPA6, IFNG, IL1B, KLRC3, MIR15A) showed significantly elevated expression $(\mathrm{FC}>1.5, \mathrm{FDR}<.05)$ (Table 3 ) and 53 genes (including $A R E G, D E F A 3$ ) were found to be significantly decreased $(\mathrm{FC}<-1.5, \mathrm{FDR}<.05)$ in the TRAIN group (Table 4). According to pathway analysis, the biological pathways that were significantly up-regulated in the TRAIN individuals after training $(\mathrm{p}<0.001)$ were those involved in inflammatory and immune response, including migration inhibitory factor (MIF) in innate immunity response $(\mathrm{p}=.00014)$, bacterial infections in normal airways $(\mathrm{p}=.0002)$, apoptosis and survival Nitric Oxide synthesis and signaling $(\mathrm{p}=.0002)$ and bacterial infections in CF airways $(\mathrm{p}=.0002)$ (data not shown). One biological pathway, translation insulin regulation of translation ( $\mathrm{p}=.00008)$, showed significantly decreased expression in TRAIN individuals after HM training (data not shown).

The TRAIN group was categorized into 10 high and 9 low responders based on percent change in their $\mathrm{rVO}_{2}$ max. Within the TRAIN group alone, 16 genes showed trending evidence of decreased expression $(\mathrm{FC}>-1.5$, FDR $<.06)$ in high vs. low responders, and one gene showed increased gene expression $(\mathrm{FC}>1.5, \mathrm{FDR}<.06)$ in the high compared to low responders group (Table 5). Biological processes that were highlighted included those involved in regulation of DNA transcription, translation and other metabolic processes. 
Table 4 Genes with decreased expression levels in active (TRAIN) vs. sedentary (CON) individuals

\begin{tabular}{|c|c|c|}
\hline \multicolumn{3}{|c|}{ After marathon training - Decreased gene expression } \\
\hline Transcript id & Gene & Fold change (FC) \\
\hline 8102787 & - & -1.5 \\
\hline 8020825 & - & -1.7 \\
\hline 8037387 & - & -1.65 \\
\hline 8136471 & - & -1.56 \\
\hline 8104490 & - & -1.56 \\
\hline 8084810 & - & -1.54 \\
\hline 8095744 & AREG & -2.42 \\
\hline 8095736 & AREG & -2.18 \\
\hline 8062444 & $\mathrm{BPI}$ & -1.74 \\
\hline 7961075 & CD69 & -1.69 \\
\hline 8149116 & DEFA3 & -2.42 \\
\hline 8149126 & DEFA3 & -2.42 \\
\hline 8149137 & DEFA3 & -2.34 \\
\hline 8084704 & EIF4A2 & -1.7 \\
\hline 8158167 & LCN2 & -1.51 \\
\hline 8086607 & LTF & -1.98 \\
\hline 7951246 & MMP8 & -1.95 \\
\hline 8012349 & PER1 & -1.59 \\
\hline 7919269 & RNU1-1 & -1.55 \\
\hline 7919349 & RNU1-1 & -1.55 \\
\hline 7973896 & RNU1-1 & -1.53 \\
\hline 7978568 & RNU1-1 & -1.53 \\
\hline 7898375 & RNU1-1 & -1.52 \\
\hline 7898411 & RNU1-1 & -1.52 \\
\hline 7912800 & RNU1-1 & -1.52 \\
\hline 7912850 & RNU1-1 & -1.52 \\
\hline 7919576 & RNU1-1 & -1.52 \\
\hline 7948894 & RNU2-1 & -1.53 \\
\hline 7938295 & RPL27A & -1.51 \\
\hline 8103975 & SLED1 & -1.62 \\
\hline 7925182 & SNORA14B & -1.5 \\
\hline 7968234 & SNORA27 & -1.52 \\
\hline 7977075 & SNORA28 & -1.56 \\
\hline 7938293 & SNORA45 & -1.6 \\
\hline 8078918 & SNORA62 & -1.86 \\
\hline 8059708 & SNORA75 & -2.03 \\
\hline 8139456 & SNORA9 & -1.55 \\
\hline 7968232 & SNORD102 & -1.67 \\
\hline 8124940 & SNORD117 & -1.67 \\
\hline 7942592 & SNORD15A & -1.53 \\
\hline 8059710 & SNORD20 & -1.93 \\
\hline 7903022 & SNORD21 & -1.69 \\
\hline
\end{tabular}

Table 4 Genes with decreased expression levels in active (TRAIN) vs. sedentary (CON) individuals (Continued)

\begin{tabular}{lll}
8159004 & SNORD24 & -1.83 \\
7948906 & SNORD27 & -1.69 \\
7948904 & SNORD28 & -1.51 \\
7948900 & SNORD30 & -1.8 \\
8030364 & SNORD34 & -1.64 \\
8159006 & SNORD36B & -1.78 \\
8005951 & SNORD42B & -1.63 \\
8005957 & SNORD4B & -1.63 \\
8023259 & SNORD58A & -1.64 \\
7964246 & SNORD59B & -1.56 \\
8122265 & TNFAIP3 & -1.61 \\
\hline
\end{tabular}

\section{Discussion}

This is the first prospective study evaluating the combination of changes in cardiac structure and function, peripheral blood cell gene expression, inflammatory markers, and potential for myocardial injury associated with training for, and performance of, a HM in previously sedentary

Table 5 Genes with significant expression in high responders* $(n=10)$ vs. low responders** $(n=9)$

\begin{tabular}{ccc}
\hline \multicolumn{2}{c}{ After marathon training - Decreased gene expression } \\
\hline Transcript id & Gene & Fold change (FC) \\
\hline 7969794 & LOC100132099 & -1.58 \\
8049530 & LRRFIP1 & -1.58 \\
7919269 & RNU1-1 & -1.89 \\
7919349 & RNU1-1 & -1.89 \\
7898375 & RNU1-1 & -1.86 \\
7898411 & RNU1-1 & -1.86 \\
7912800 & RNU1-1 & -1.86 \\
7912850 & RNU1-1 & -1.86 \\
7919576 & RNU1-1 & -1.86 \\
7973896 & RNU1-1 & -1.85 \\
7978568 & RNU1-1 & -1.85 \\
7897801 & RNU5E & -1.89 \\
7920873 & SNORA42 & -3.49 \\
8117018 & - & -1.86 \\
7932635 & - & -1.63 \\
7965150 & - & -1.62 \\
\hline
\end{tabular}

After marathon training - increased gene expression

\begin{tabular}{l} 
Transcript id $\quad$ Gene \\
\hline 8005225 \\
LOC 162632
\end{tabular}


subjects. The decreases in blood pressure, resting heart rate, and body fat percentage, and the increases in $\mathrm{VO}_{2}$ max, observed in our TRAIN group over the course of the program are consistent with previously well-documented responses to exercise training $[15,16]$ and validate the training effect in our HM participants. More interesting is the effect of $\mathrm{HM}$ training on biomarkers related to myocardial injury, cardiovascular structural changes by echocardiogram, serum lipids, gene expression and serum inflammatory markers.

In our study, LV mass and wall thickness, and LV end-diastolic and stroke volumes increased significantly after 16 weeks of training. The $\mathrm{E}$ to $\mathrm{A}$ ratio also decreased throughout training, consistent with a decrease in diastolic relaxation commonly seen in hypertrophied ventricles. These echocardiographic changes have been well-established and recently described to occur in endurance athletes in as little as 90 days [17-19]. This phenomenon, known as the "athlete's heart," is thought to be due to a long-sustained high cardiac output resulting in a volume-loaded heart that can lead to an increase in LV cavity, LV mass, and wall thickness [20-22].

Both TRAIN and CON groups had the same inclusion criteria, which enabled us to maximize the comparison after training. When comparing gene expression profiles in blood collected before training in TRAIN versus CON men, 4 genes showed significant changes in gene expression $(\mathrm{FC}=(1.5-1.7) \mathrm{FDR}<.05)$ suggesting that the two groups were fairly similar before training. Through pathway analysis, the biological pathways that were significantly up regulated in the TRAIN individuals after training were those primarily involved in the body's inflammatory and immune response. In looking at the specific genes involved of the 53 genes that showed significantly decreased expression in TRAIN individuals after training, Amphiregulin (AREG) was most significantly decreased $(\mathrm{FC}=-2.42)$. The protein coded by AREG is a member of the epidermal growth factor family. AREG has both growth factor and inhibitory properties (NCBI, PubMed Gene ID:374). Changes in AREG expression in human neutrophils have been related to exercise in previous studies. For example, following 30 minutes of aerobic exercise, AREG was shown to increase by 3.4 fold [23]. Although the change is in the opposite direction of the change found in the current study this could be due to the fact that Radom-Aizik et al. [23] examined gene expression changes following acute exercise, whereas our focus is on gene expression changes due to long term, consistent exercise.

Another gene significantly down-regulated in the TRAIN individuals; defensin, alpha 3, neutrophil-specific (DEFA3) also has a role in both the immune and inflammatory response (NCBI, PubMed Gene ID: 1668). A previous study linked increased expression of DEFA3 at both the
mRNA and protein level to inflammatory diseases [24]. It is well established that regular exercise helps reduce the amount of total body inflammation and therefore helps prevent the induction of many chronic inflammatory diseases including atherosclerosis. A decrease in total body inflammation is usually associated with a decrease in C-reactive protein, which was not observed in our study. This data suggests that perhaps gene expression changes associated with a decrease in inflammation occur prior to changes in cardiac biomarkers or that subjects with normal CRP at baseline are less likely to show a measurable response to exercise associated with changes in gene expression. Perhaps with longer training, or elevated baseline levels of CPR, similar changes in the cardiac biomarkers would also be seen.

When comparing the TRAIN high responders and low responders, several gene expression differences were noted. Two genes that showed increased expression in high responders before training included FGFBP2 $(\mathrm{FC}=1.76)$ and $C D 160$ (FC = 1.55). Both are involved in immune response (NCBI, PubMED Gene ID: 11126).

Of the genes that showed decreased expression in high responders after exercise of particular note is LRRFIP1 $(\mathrm{FC}=-1.57)$. LRRFIP1 has been identified as having a role as both a regulator of toll-like receptor pathway signaling and transcriptional repression. Its expression has been previously shown to be induced by nicotine and proinflammatory cytokines [25].

\section{Conclusions}

In summary, in previously sedentary individuals, intensive training for a HM over 17 weeks leads to increased cardiac and hemodynamic performance that is not associated with longitudinal changes in known cardiac biomarkers. Elevated cTnT occurred in only 1 of 16 subjects who completed the HM. Training is also associated with changes in blood gene expression that reflects the anabolic state of the body, as well as changes in the body's immune and inflammatory response that could be more sensitive precursors to changes in serum biomarkers. Whereas some genes involved in the immune/inflammatory response showed increased expression, others showed decreased expression implying that exercise brings about changes that both suppress aspects of the immune system while stimulating others. Genes with changes in gene expression as response to HM-training include AREG, DEFA3, CD160, GPR56, KLRC3, and LRRFIP1. The results presented here are from a single sample and do not withstand conservative corrections for multiple comparisons. The study's sample is limited to men and therefore cannot necessarily be generalized to the broader population which could include females However, they generate interesting hypotheses about a possible role of genes involved in immunity and inflammation in 
responding to intensive exercise training, which bear examination in other data sets.

\section{Competing interests}

The authors declare that they have no competing interests.

\section{Authors' contributions}

DDV: study design, RNA extraction, gene expression and pathway analysis, manuscript preparation. GC: echocardiogram data collection, cardiac biomarker analysis, manuscript preparation. MS: ascertainment, data collection. RJM: study design, cardiac biomarker analysis, manuscript editing $\mathrm{KJ}$ : ascertainment, data collection. LN: microarray and gene expression analysis. AP: data collection, study design, editing. DS: study design, gene expression analysis, manuscript editing. PJGC: study design, manuscript editing. ER statistical analysis, gene expression and pathway analysis, manuscript editing. All authors read and approved the final manuscript.

\section{Acknowledgements}

The authors wish to thank Dr. Martin Bilsker, Director of the Echocardiography lab in Jackson Memorial Hospital, Miami, FL, for reading the echocardiograms in this study. Also special thanks to Diego Duque and Philips for the use of their Philips HD 11 Digital Ultrasound Machine. Thanks to the laboratory and analytical staff of the Hussman Institute for Human Genomics for their support in particular Dr. Demet Tekin. Dr. Myerburg is supported in part by a grant from the Fondation Leducq, Paris, France (Trans-Atlantic Network of Excellence "Alliance Against Sudden Cardiac Death"). Research conducted in this paper was funded in part by funds from Florida's Office of Tourism, Trade, and Economic Development.

\section{Author details}

'John P. Hussman Institute for Human Genomics, University of Miami, Miami, FL, USA. '2Department of Kinesiology and Sports Science, University of Miami, Miami, FL, USA. ${ }^{3}$ Department of Medicine, Miller School of Medicine, University of Miami, Miami, FL, USA. ${ }^{4}$ Department of Physiology, Miller School of Medicine, University of Miami, Miami, FL, USA. ${ }^{5}$ Dr. John T. Macdonald Foundation Department of Human Genetics, University of Miami, Miami, FL, USA. ${ }^{6}$ Department of Epidemiology \& Public Health, Miller School of Medicine, University of Miami, Miami, FL, USA.

Received: 1 April 2013 Accepted: 6 February 2014 Published: 19 February 2014

\section{References}

1. Bernadet P: Benefits of physical activity in the prevention of cardiovascular diseases. J Cardiovasc Pharmacol 1995, 25(Suppl 1):S3-S8.

2. Giannuzzi P, Mezzani A, Saner H, Bjornstad H, Fioretti P, Mendes M, CohenSolal A, Dugmore L, Hambrecht R, Hellemans I, McGee H, Perk J, Vanhees L, Veress G, Working Group on Cardiac Rehabilitation and Exercise Physiology. European Society of Cardiology: Physical activity for primary and secondary prevention. Position paper of the Working Group on Cardiac Rehabilitation and Exercise Physiology of the European Society of Cardiology. Eur J Cardiovasc Prev Rehabil 2003, 10:319-327.

3. Physical activity and cardiovascular health. NIH consensus development panel on physical activity and cardiovascular health. JAMA 1996, 276:241-246. http://jama.jamanetwork.com/article.aspx?articleid=405583.

4. Siegel AJ, Lewandrowski EL, Chun KY, Sholar MB, Fischman AJ, Lewandrowski KB: Changes in cardiac markers including B-natriuretic peptide in runners after the Boston marathon. Am J Cardiol 2001, 88:920-923.

5. Briviba K, Watzl B, Nickel K, Kulling S, Bos K, Haertel S, Rechkemmer G, Bub A: A half-marathon and a marathon run induce oxidative DNA damage, reduce antioxidant capacity to protect DNA against damage and modify immune function in hobby runners. Redox Rep 2005, 10:325-331.

6. Neilan TG, Januzzi JL, Lee-Lewandrowski $\mathrm{E}$, Ton-Nu TT, Yoerger DM, Jassal DS, Lewandrowski KB, Siegel AJ, Marshall JE, Douglas PS, Lawlor D, Picard $\mathrm{MH}$, Wood MJ: Myocardial injury and ventricular dysfunction related to training levels among nonelite participants in the Boston marathon. Circulation 2006, 114:2325-2333.

7. Neilan TG, Yoerger DM, Douglas PS, Marshall JE, Halpern EF, Lawlor D, Picard MH, Wood MJ: Persistent and reversible cardiac dysfunction among amateur marathon runners. Eur Heart J 2006, 27:1079-1084.
8. Fortescue EB, Shin AY, Greenes DS, Mannix RC, Agarwal S, Feldman BJ, Shah MI, Rifai N, Landzberg MJ, Newburger JW, Almond CS: Cardiac troponin increases among runners in the Boston Marathon. Ann Emerg Med 2007, 49:137-143. 143.e1.

9. Bouchard C, Sarzynski MA, Rice TK, Kraus WE, Church TS, Sung YJ, Rao DC, Rankinen T: Genomic predictors of the maximal $\mathrm{O}$ uptake response to standardized exercise training programs. J App/ Physiol 2011, 110:1160-1170.

10. Shave R, George KP, Atkinson G, Hart E, Middleton N, Whyte G, Gaze D, Collinson PO: Exercise-induced cardiac troponin T release: a meta-analysis. Med Sci Sports Exerc 2007, 39:2099-2106.

11. Schiller NB, Shah PM, Crawford M, DeMaria A, Devereux R, Feigenbaum H, Gutgesell H, Reichek N, Sahn D, Schnittger I: Recommendations for quantitation of the left ventricle by two-dimensional echocardiography. American society of echocardiography committee on standards, subcommittee on quantitation of two-dimensional echocardiograms. J Am Soc Echocardiogr 1989, 2:358-367.

12. Alpert JS, Thygesen K, Antman E, Bassand JP: Myocardial infarction redefined-a consensus document of the joint European society of cardiology/American college of cardiology committee for the redefinition of myocardial infarction. J Am Coll Cardiol 2000, 36:959-969.

13. Tusher VG, Tibshirani R, Chu G: Significance analysis of microarrays applied to the ionizing radiation response. Proc Natl Acad Sci USA 2001, 98:5116-5121.

14. Benjamini $Y$, Hochberg $Y$ : Controlling the false discovery rate: a practical and powerful approach to multiple testing. Ann Stat 1995, 57:289-300.

15. Kelley GA, Kelley KS: Efficacy of aerobic exercise on coronary heart disease risk factors. Prev Cardiol 2008, 11:71-75.

16. Kaplan NM: Non-drug treatment of hypertension. Ann Intern Med 1985 102:359-373.

17. Morganroth J, Maron BJ, Henry WL, Epstein SE: Comparative left ventricular dimensions in trained athletes. Ann Intern Med 1975, 82:521-524.

18. Baggish AL, Wang F, Weiner RB, Elinoff JM, Tournoux F, Boland A, Picard $\mathrm{MH}$, Hutter AM Jr, Wood MJ: Training-specific changes in cardiac structure and function: a prospective and longitudinal assessment of competitive athletes. J App/ Physio/ 2008, 104:1121-1128.

19. Maron BJ: Structural features of the athlete heart as defined by echocardiography. J Am Coll Cardiol 1986, 7:190-203.

20. Khamis RY, Mayet J: Echocardiographic assessment of left ventricular hypertrophy in elite athletes. Heart 2008, 94:1254-1255.

21. Naylor LH, George K, O'Driscoll G, Green DJ: The athlete's heart: a contemporary appraisal of the 'Morganroth hypothesis'. Sports Med 2008, 38:69-90.

22. Mihl C, Dassen WR, Kuipers H: Cardiac remodelling: concentric versus eccentric hypertrophy in strength and endurance athletes. Neth Heart $J$ 2008, 16:129-133.

23. Radom-Aizik S, Kaminski N, Hayek S, Halkin H, Cooper DM, Ben-Dov I: Effects of exercise training on quadriceps muscle gene expression in chronic obstructive pulmonary disease. J Appl Physiol 2007, 102:1976-1984

24. Froy O, Sthoeger ZM: Defensins in systemic lupus erythematosus. Ann N Y Acad Sci 2009, 1173:365-369.

25. Arakawa R, Bagashev A, Song L, Maurer K, Sullivan KE: Characterization of LRRFIP1. Biochem Cell Biol 2010, 88:899-906.

doi:10.1186/2052-1847-6-6

Cite this article as: Vance et al:: Cardiac performance, biomarkers and gene expression studies in previously sedentary men participating in half-marathon training. BMC Sports Science, Medicine, and Rehabilitation 2014 6:6 